Daniel Jacob

\title{
Der Nationalsozialismus, Richard Rorty und die Möglichkeiten rationaler Verständigung1
}

\section{Grenzen der Verständigung}

Der Nationalsozialismus ist auf den ersten Blick ein Paradebeispiel dafür, dass rationale Verständigung im Angesicht ideologischen Beharrens zum Scheitern verurteilt ist. In ihrem völkischen Wahn waren die Nationalsozialisten weder von den demokratischen Kräften innerhalb Deutschlands noch vom zunehmend besorgten Ausland »zur Vernunft zu bringen«. Im Gegenteil radikalisierte sich im Zuge der nationalsozialistischen Herrschaft ihre Ideologie sogar noch weiter, mit katastrophalen Folgen. Nach Ende des Krieges beobachtet Hannah Arendt bei einer Reise durch das stark zerstörte Deutschland eine weitverbreitete moralische Indifferenz, eine Unfähigkeit, das Ausmaß der eigenen Verbrechen zu erfassen. ${ }^{2}$ Das nationalsozialistische Deutschland, so scheint es, befand sich in einem Geisteszustand, der eine unüberwindbare Kluft zum humanistisch-liberalen Denken bis über das Ende des Krieges hinaus offenbarte.

Für den amerikanischen Neo-Pragmatisten Richard Rorty zeigt sich hier eine kommunikative Barriere, die weniger dramatisch immer schon unseren Alltag bestimmt. Geprägt von unserem sozialen Umfeld, so Rorty, nähern wir uns der Welt aus der Perspektive eines spezifischen »Vokabulars «, sehen diese immer durch den Filter kultureller Konventionen. Rationale Verständigung, der Austausch von Argumenten mit Bezug auf gemeinsam geteilte Prämissen, sei über die Grenzen von Vokabularen hinweg nicht möglich. ${ }^{3}$ Aufmerksamkeit hat Rorty mit diesen Argumenten insbesondere in der Debatte um den universellen Geltungsanspruch von Menschenrechten erregt. So wünschenswert Menschenrechte aus liberaler Perspektive auch erscheinen mögen, seien sie doch bloß Teil eines kontingenten, spezifisch-

1 Für hilfreiche Kommentare und Anregungen möchte ich insbesondere Jan Brezger, Nina Engwicht, Eva Hausteiner, Bernd Ladwig, Thorsten Thiel, Christian Volk sowie den zwei anonymen Gutachtern des »Leviathan " danken. Zudem danke ich der Deutschen Forschungsgesellschaft für die Förderung des Sonderforschungsbereichs 700 » Governance in Räumen begrenzter Staatlichkeit ", in dessen Kontext dieser Text entstanden ist.

2 Vgl. Arendt 1950, S. 342.

3 Für einen Überblick zum Denken Rortys siehe Auer 2004 und Iser 2008, S. 51-65. Zu seiner Rolle im Kontext des amerikanischen Pragmatismus siehe Talisse 2007, S. 1-26, und Bernstein 2010 a, S. 13-15. Zur Person Rortys siehe Rorty 2000 b; Bernstein 2008; Gross 2008. Zur Bandbreite der Rezeption von Rorty siehe Ramberg 2000, S. 127-129. Einen Zugang ganz eigener Art bietet das Online-Archiv der University of California Irvine, das den digitalen Nachlass von Rorty verwaltet; siehe University of California Irvine 2010.

Leviathan, 40. Jg., 1/2012, S. $109-128$ 
westlichen Vokabulars; kein noch so ausgefeiltes Argument könne ihren besonderen Stellenwert über die Grenzen dieses Vokabulars hinaus begründen. ${ }^{4}$ Wiederholt greift Rorty dabei auf das Beispiel des Nationalsozialismus zurück. Mit besonderer Deutlichkeit zeige sich hier die Unmöglichkeit, zwischen verschiedenen Vokabularen zu vermitteln und so über deren Grenzen hinweg zu einer rationalen Verständigung zu gelangen: »Das liegt daran, dass wir lieber sterben als unschuldige jüdische Kinder töten würden, während die Nazis (wie wir, um ein extremes Beispiel zu nennen, sagen wollen) lieber sterben würden, als den Führer zu verraten, indem sie die unschuldigen Judenkinder nicht umbringen $" .{ }^{5}$ Dabei profitiert Rorty ersichtlich von der suggestiven Kraft seines Beispiels: Konfrontiert mit den für uns tatsächlich unvorstellbaren Gräueltaten des NS-Regimes scheint seine Position intuitiv plausibel. Tatsächlich wirkt der Versuch einer rationalen Verständigung mit überzeugten Nazis auf den ersten Blick geradezu grotesk.

Und doch möchte ich argumentieren, dass Rorty vorschnell die Möglichkeiten rationaler Verständigung verwirft und damit zu fragwürdigen politischen Schlussfolgerungen gelangt. Der Grund hierfür liegt in seiner unzureichenden Ausdifferenzierung des für ihn so zentralen Begriffs des Vokabulars, aus der seine sehr eng gefasste Vorstellung von rationaler Verständigung herrührt. Wie schon erwähnt, argumentiert Rorty, dass über die Grenzen von Vokabularen hinweg rationale Verständigung nicht möglich sei, wohl aber innerhalb eines Vokabulars. Dabei geht er im Kern von einer Vorstellung rationaler Verständigung aus, wie sie auch den hier angestellten Überlegungen zugrunde liegt: Rationale Verständigung bezeichnet demnach den Austausch von Argumenten mit Bezug auf gemeinsam geteilte Prämissen oder, anders ausgedrückt, die Bereitschaft, Geltungsansprüche mit Bezug auf eben diese gemeinsam geteilten Prämissen anzuerkennen. ${ }^{6}$ Innerhalb eines Vokabulars ist diese Art der Verständigung nach Rorty möglich, da hier die notwendige Gemeinsamkeit der Prämissen vorhanden ist; bei Verständigungsversuchen über die Grenzen von Vokabularen hinweg fehlt sie jedoch und ist rationale Verständigung daher unmöglich. Da Rorty letztlich die vokabularübergreifende Verständigung, beispielsweise über den Gehalt der Menschenrechte, für ausschlaggebend hält, neigt er dazu, rationale Verständigung in Gänze zu verwerfen. So etwa in seinem berühmt gewordenen Diktum, anrührenden Erzählungen wie Onkel Toms Hütte komme eine bei weitem größere Bedeutung für die Verbreitung der Menschenrechte zu als noch der ausgefeiltesten moralphilosophischen Begründung, die letztlich eben doch nur ein zum Scheitern verurteilter Versuch rationaler Verständigung bleibe. ${ }^{7}$

Rortys Argument über die Vergeblichkeit rationaler Verständigung hängt dabei direkt von seinen Überlegungen zum Begriff des Vokabulars ab, basiert seine Skepsis doch auf der Vorstellung miteinander inkommensurabler Vokabulare. Der hier gemachte Vorschlag einer weiteren Ausdifferenzierung des Vokabularbegriffs will ge-

\author{
4 Vgl. Rorty 2000 a. \\ 5 Rorty 2001 c, S. 109. \\ 6 Rorty 2001 a, S. 165; Rorty, Mendieta 2006, S. 70. \\ 7 Rorty 2000 a, S. 265-267.
}


nau diese Vorstellung in Frage stellen. Zwar bleibt es weiterhin möglich, dass rationale Verständigung aufgrund mangelnder geteilter Prämissen an den Grenzen von Vokabularen scheitert, doch zeigt sich bei einem dynamischeren Verständnis von Vokabularen, dass der von Rorty zugestandene, doch leichthin verworfene Fall der rationalen Verständigung zwischen Vokabularen sehr viel mehr Potential birgt. Ein dynamischeres Verständnis legt nahe, dass es zwischen den meisten Vokabularen ausreichende Überschneidungen gibt, um rationale Verständigung zu ermöglichen. Insofern, als diese Überschneidungen dann wiederum als ein gemeinsames Vokabular begriffen werden können, wird die von Rorty zum Sonderfall erklärte rationale Verständigung innerhalb eines gemeinsamen Vokabulars ganz im Gegenteil zum Regelfall - und scheint mithin eine stärkere Beachtung der Möglichkeiten rationaler Verständigung angezeigt.

Bei genauerem Hinsehen bietet sich gerade das von Rorty gewählte Beispiel des Nationalsozialismus an, um diese Überlegungen zu verdeutlichen. In seinen radikalsten Ausprägungen lässt sich der Nationalsozialismus tatsächlich als ein Vokabular begreifen, dessen Überlappungen mit anderen Vokabularen für rationale Verständigung nicht ausreichend waren. In diesem Sinne sind die Verbrechen der Nationalsozialisten für uns wahrlich unverständlich, da wir sämtliche der relevanten moralischen und pseudo-biologischen Hintergrundannahmen nicht teilen. Zugleich lässt sich aber selbst noch am Beispiel des Nationalsozialismus zeigen, dass es sich bei diesem Scheitern rationaler Verständigung um den Endpunkt eines Prozesses handelt, im Zuge dessen Überlappungen mit anderen Vokabularen und damit die Voraussetzung für rationale Verständigung systematisch eliminiert wurden. Nimmt man diesen Prozess und damit den dynamischen Charakter von Vokabularen stärker in den Blick, so zeigen sich gerade hier die vielfältigen Möglichkeiten rationaler Verständigung, die Rorty trotz einiger Andeutungen insgesamt aus dem Blick verloren zu haben scheint.

Um diese Überlegungen zu verdeutlichen, beginne ich mit einer Darstellung neuerer historischer Studien zum moralischen Selbstverständnis der Anhänger des Nationalsozialismus (II). Interessant für die Ausgangsfrage ist hier die von Harald Welzer und Raphael Gross vorgeschlagene Perspektive, diese gerade nicht als amoralisch zu verstehen. Vielmehr, so Welzer und Gross, müsse man die nationalsozialistische Ideologie als einen für breite Bevölkerungsschichten wirksamen normativen Referenzrahmen, eben als moralisches System, verstehen. In diesem Sinne handelten auch die an "Judenaktionen « beteiligten Soldaten moralisch, so sehr die diesem Handeln zugrunde liegende Moral uns auch verwerflich und schon mit moralischen Grundprinzipien wie dem der Unparteilichkeit unvereinbar erscheint. ${ }^{8}$ Diese historischen Untersuchungen verknüpfe ich mit einer Diskussion des Vokabularbegriffs bei Rorty (III). Wie schon angedeutet, steht dabei im Zentrum die Frage, inwiefern die historischen Studien zur Entstehung der »nationalsozialistischen Moral « Hinweise für eine Klärung und weitere Ausdifferenzierung des Vokabularbegriffs bieten können. Schließlich diskutiere ich im abschließenden Teil des Textes, welche politischen Konsequenzen sich aus der Überarbeitung des Vokabularbegriffs ergeben

8 Zum Prinzip der Unparteilichkeit siehe Barry 1995 sowie überblickshaft Özmen 2008.

Leviathan, 40. Jg., 1/2012 
(IV). Insbesondere weise ich dabei auf die Notwendigkeit der institutionellen Absicherung rationaler Verständigung in Form eines Pluralismus von Vokabularen hin.

\section{Die partikulare Moral des Nationalsozialismus}

Harald Welzer und Raphael Gross eint in ihren historischen Studien ${ }^{9}$ die Herangehensweise, den Nationalsozialismus auch als ein moralisches Wertesystem mit handlungsleitender Funktion zu verstehen. Beide schlagen vor, dieses Wertesystem im Sinne einer "partikularen " Moral zu deuten: Wird Moral in philosophischen Diskursen üblicherweise als Ausdruck des Standpunktes der Unparteilichkeit verstanden, so definiert sich die partikulare nationalsozialistische Moral gerade durch den Ausschluss bestimmter Gruppen, in erster Linie der Juden, aus der Sphäre moralischer Bedeutsamkeit. In diesem Sinne beschreibt Welzer die nationalsozialistische Moral als geprägt durch »die Vorstellung erstens von einer absoluten Ungleichheit von Menschen, die aus Sicht der Akteure wissenschaftlich begründet war, und zweitens die Setzung, dass diese Ungleichheit eine Bedrohung für die nach rassistischen Kriterien höherwertige Gruppe von Menschen bedeutete, der man um des eigenen Überlebens willen begegnen musste « ${ }^{10}$

Welzer bedient sich dieser Perspektive, um eine Antwort auf die schon im Titel seiner Überlegungen gestellte Frage zu finden, »wie aus ganz normalen Menschen Massenmörder werden «. Ausgangspunkt ist dabei für ihn der empirische Befund, dass nur ein geringer Teil der nationalsozialistischen Täter psychologische Anomalien aufwiesen. Bei nur etwa zehn Prozent, so Welzer mit Verweis auf entsprechende Untersuchungen, habe der Anteil derjenigen gelegen, die sich am Töten aus eigenem inneren Antrieb und häufig mit unverhohlener Lust beteiligten. Eine Erklärung, die von der individualpsychologischen Ebene ausgeht, scheide daher aus. Die riesige und dabei erschreckend effiziente Tötungsmaschinerie der Nationalsozialisten habe außerdem auch nicht, wie im Nachhinein von vielen Tätern als Schutzbehauptung in juristischen Verhandlungen gerne angeführt, auf dem Zwang militärischer Hierarchien (»Befehlsnotstand «) basiert. Vielmehr, so Welzers zentrale These, habe das nationalsozialistische Regime in einem schrittweisen Prozess eine Verschiebung des normativen Referenzrahmens bewirkt, die es den Tätern schließlich rechtfertigbar und gar als moralisch geboten erscheinen ließ, sich an Erschießungsaktionen zu beteiligen. ${ }^{11}$

Dabei ist jedoch noch einmal zu unterscheiden zwischen der allgemeinen Verschiebung des normativen Referenzrahmens durch das NS-Regime und der besonderen Situation der unmittelbar an Tötungsaktionen hinter der Front beteiligten Täter. Die allgemeine Verschiebung des Referenzrahmens setzt nach Welzer spätestens in dem Moment ein, in dem durch die Nürnberger Rassengesetze die antisemitische Ideologie der Nationalsozialisten offizielle Politik des Staates und als

9 Welzer 2007; Gross 2010.

10 Welzer 2007, S. 31.

11 Vgl. ebd., S. 7-17. 
solche in positives Recht umgesetzt wird. Damit, so Welzer, kommt es zu einer Ausgliederung der Juden aus der nun in moralischer Hinsicht für einzig relevant erklärten Wir-Gruppe der »Volksdeutschen«. Die rasante Abfolge antisemitischer Diskriminierungen verändert das normative Gefüge und lässt dann schließlich auch das »Verschwinden « von Juden als kaum noch problematisch erscheinen. ${ }^{12} \mathrm{Im} \mathrm{Ge}-$ genteil, im Anschluss an die Studien von Daniel Goldhagen und Götz Aly geht Welzer davon aus, dass die Ausgrenzung der Juden infolge einer pervertierten Logik selbst noch deren eigene Rechtfertigung lieferte, schien doch die geschehene Ausgrenzung die herbeifantasierte Überlegenheit der deutschen Volksgemeinschaft wiederum zu bestätigen. Mit jedem »verschwundenen « jüdischen Nachbarn konnte sich der arisch gefestigte Deutsche seiner eigenen sozialen Stellung ein wenig sicherer sein - und vielleicht auch noch einen günstigen Mantel erstehen. ${ }^{13}$

An dieser Stelle bieten die Studien von Raphael Gross eine hilfreiche Ergänzung zu jenen von Welzer. Wo bei Welzer die bisher ausgeführten Überlegungen vor allem dazu dienen, das Verhalten der unmittelbar an Mordaktionen beteiligen Täter zu erklären, will Gross die alltägliche Verbreitung der nationalsozialistischen Moral in der Breite der deutschen Bevölkerung nachzeichnen. ${ }^{14}$ Dabei argumentiert er, dass die nationalsozialistische Führung gezielt an bestehende moralische Vorstellungen anknüpfte, um diese in ihrem Sinne umzudeuten und sich so eine nachhaltige $\mathrm{Zu}$ stimmung breiter Bevölkerungsschichten zu sichern. ${ }^{15}$ Am Begriff der Schande zeigt Gross auf, wie es durch Propaganda etwa in Form des Films "Jud Süß « recht erfolgreich gelang, diesen als »Rassenschande « im Sinne der eigenen Ideologie umzudeuten. ${ }^{16}$ Auch Begriffe wie Ehre oder Treue wurden an die Bedürfnisse des Regimes angepasst und auf die Figur des Führers zugeschnitten. Wahnwitzig etwa die Umformulierung von Kants kategorischem Imperativ durch den Reichsrechtsführer und späteren Generalgouverneur im besetzten Polen, Hans Frank: »Handle so, dass der Führer, wenn er von deinem Handeln Kenntnis hätte, dieses Handeln billigen würde «. ${ }^{17}$

Welzer geht jedoch noch einen Schritt weiter und möchte erklären, wie diese allgemeine Verschiebung des normativen Referenzrahmens derart konkret wirksam werden konnte, dass sie bis dahin nicht durch Gewalttaten auffällig gewordene Menschen dazu brachte, Hunderte von Menschen mit eigener Hand zu töten. Was das historische Material betrifft, bezieht er sich dabei insbesondere auf die Untersuchungen von Christopher Browning, der in seinem Buch » Ganz normale Männer. Das Reserve-Polizeibataillon 101 und die 'Endlösung` in Polen «18 auf Basis von Protokollen späterer Gerichtsverhandlungen detailliert die Handlungen eines an

12 Ebd., S. 48-67.

13 Ebd., S. 250-252; vgl. auch Goldhagen 2000 und Aly 2005.

14 Vgl. hierzu auch Koonz 2004.

15 Gross 2010, S. 18-20.

16 Ebd., S. 33-35.

17 Ebd., S. 256.

18 Siehe Browning 1999.

Leviathan, 40. Jg., 1/2012 
zahlreichen "Judenaktionen " beteiligten Polizeibataillons und insbesondere auch die Selbstwahrnehmung der an den Erschießungen beteiligten Täter rekonstruiert hat.

Dabei zeigt sich, wie diese Täter schrittweise eine »Tötungsmoral « (Welzer) annehmen, die sich als nochmalige Radikalisierung der auf Ausschluss gründenden nationalsozialistischen Moral verstehen lässt. So gibt es bei den ersten Erschießungsaktionen noch einige Offiziere und auch Angehörige der Mannschaften, die sich auf unterschiedliche Weise der Teilnahme an diesen Aktionen entziehen. Manche bitten ganz offiziell um eine Freistellung von dieser Aufgabe, andere geben sich beschäftigt oder verstecken sich. ${ }^{19}$ Bei einigen wenigen war dies in humanistischen Einstellungen begründet, hinzu kamen bürgerliches Ehrgefühl, christliche Erziehung oder auch nur ein militärisches Selbstverständnis, für den Kampf und nicht für solch "mindere " Aufgaben ausgebildet worden zu sein. Bezeichnend ist etwa der von Browning erwähnte Fall eines älteren Hamburger Geschäftsmannes, der als Reaktion auf den Befehl zur Teilnahme an einer Erschießungsaktion erklärt, als Hanseat und Geschäftsmann nicht für derlei Aktionen zur Verfügung zu stehen. Seine Erklärung wird akzeptiert, und er wird einer anderen Aufgabe zugeteilt. Binnen weniger Monate wird er dann auf eigenen Wunsch wieder in seine Heimatstadt Hamburg zurückversetzt. ${ }^{20}$ Welzer weist darauf hin, dass sich seitens der befehlsgebenden Offiziere zu Beginn der Erschießungsaktionen noch eine gewisse Unsicherheit im Umgang mit der neuen Aufgabe, massenweise Juden zu erschießen, zeigt. Dies eröffnet auf individueller Ebene Spielräume, die es ermöglichen, sich nicht an den Erschießungen zu beteiligen. Die meisten jedoch töten. ${ }^{21}$

Je länger das Töten anhält, je mehr es zur Routine wird, umso weniger problematisch erscheint den Angehörigen des Polizeibataillons ihre Aufgabe. Welzer macht eine Reihe von sich gegenseitig verstärkenden Faktoren aus, die diese Normalisierung der massenhaften Tötung von Menschen möglich machen: Erstens begreifen die Männer das Töten bald als »Arbeit «, die es wie jede andere Arbeit möglichst professionell auszuführen gilt. Das Leiden der Opfer rückt dabei schnell in den Hintergrund, entscheidender werden nun technische Fragen der optimalen Tötung, die bisweilen auch in Anwesenheit der Opfer im Rahmen von »Fachgesprächen « erörtert werden. ${ }^{22}$ Zweitens zeigt schon die Unverfrorenheit, im Angesicht des nächsten Opfers dessen bestmögliche Erschießung zu diskutieren, wie sehr die Opfer bewusst entindividualisiert wurden. Die so gezielt herbeigeführte Distanz lässt die Opfer nur noch als eine lange Reihe zu bearbeitender »Objekte " erscheinen. ${ }^{23}$ Wie wirksam diese Distanzierung funktionierte, zeigt sich besonders deutlich dort, wo sie versagt, wenn etwa die Täter mit Erschrecken feststellen müssen, dass

19 Ebd., S. 108.

20 Ebd., S. 109 f.

21 Ebd., S. 241.

22 Welzer 2007, S. 129, 202 f.

23 Ebd., S. 262. 
eines ihrer Opfer deutsch spricht. ${ }^{24}$ Drittens erlaubt es ein hohes Maß an Arbeitsteilung den Tätern, ihr Handeln in ihrer Selbstwahrnehmung in einen Bereich jenseits ihrer individuellen Verantwortung zu verschieben. Bezeichnend ist hier, dass viele derjenigen, die »nur " die Opfer aus ihren Häusern geholt haben oder diese »nur « von der Flucht abgehalten haben, sich selbst nicht eigentlich als Täter verstanden. ${ }^{25}$ Besonders deutlich wird die Funktion dieser Arbeitsteilung, wenn sogenannte "Hilfswillige " aus Litauen oder der Ukraine unter Todesdrohungen zur Durchführung von selbst nach damaligen Maßstäben besonders unangenehmen Tötungsaktionen gezwungen werden. ${ }^{26}$

Vor allem aber entwickelt sich der Mikrokosmos des eigenen Bataillons bald zum immer exklusiveren normativen Bezugsrahmen. Zwar wird anfangs noch ein erheblicher Aufwand betrieben, um die Erschießungen wenigstens dem Anschein nach in einen militärischen oder polizeilichen Kontext einzubetten und so an bestehende normative Vorstellungen anzuknüpfen. ${ }^{27}$ Spätestens mit der Ausweitung des Kreises der Opfer auf Kinder gerät diese Umdeutung bestehender Normen dann jedoch an ihre Belastungsgrenze, ist nun aber auch gar nicht mehr notwendig. ${ }^{28}$ Die Täter haben nun oft genug gesehen, wie andere getötet haben, haben selbst getötet und schließlich mit einer gewissen Überraschung festgestellt, dass dies möglich ist, »ohne dass dadurch für einen die Welt untergeht «. ${ }^{29}$ Tatsächlich wird das Töten im sozialen Binnen-Kontext der Einsatzgruppen ja nicht nur geduldet, sondern als moralische Pflicht gegenüber Deutschland und »den Lieben daheim " gar gefordert. Und, dies ist entscheidend, je länger die Männer in ihrem mörderischen Treiben fortschreiten, umso mehr verblassen frühere Vorstellungen moralisch richtigen Handelns. Es kommt zu einer Situation, in der »die Männer unter sich sind und keine Personen jenseits ihrer Gruppe mehr existieren, die den Referenzrahmen der Gruppe in Frage stellen könnten «.30

Dass die allermeisten Täter sich in dieser Situation tatsächlich als moralisch Handelnde wähnten, kommt in zwei von Welzer beschriebenen Phänomenen besonders deutlich zum Ausdruck. Zum einen beschreiben viele der Täter in Briefen an ihre Familien in der Heimat ganz offen und mit unverkennbarem Stolz ihre Taten; etwa wenn einer der Männer in einem Brief an seine Frau schreibt: »Bei den ersten Wagen [mit Opfern] hat mir etwas die Hand gezittert, als ich geschossen habe, aber man gewöhnt [sich an] das. Beim zehnten Wagen zielte ich schon ruhig und schoß sicher auf die vielen Frauen, Kinder und Säuglinge. ... Der Tod, den wir ihnen gaben, war ein schöner, kurzer Tod «. ${ }^{31}$ Zum anderen weist Welzer darauf hin, dass es vielen

24 Ebd., S. 155.

25 Ebd., S. 120-122.

26 Ebd., S. 130.

27 Ebd., S. $127 \mathrm{f}$.

28 Ebd., S. 174.

29 Ebd., S. 124.

30 Ebd., S. 115 f.

31 Ebd., S. 185. 
Tätern für die moralische Deutung des eigenen Handelns darauf ankam, sich von den » Gewaltexzessen « anderer abzugrenzen. So wie Himmler in seiner berüchtigten Rede im damaligen Posen 1943 betonte, wie wichtig es sei, auch angesichts solcher Aufgaben wie der »Endlösung « »anständig geblieben zu sein ", legen auch die einzelnen Täter Wert darauf, ihren Opfern »unnötige Qualen « erspart zu haben. ${ }^{32}$ Gerade hier wird die besondere Erklärungskraft der Perspektive von Welzer deutlich: Selbst im Angesicht massenhafter Tötungen ist es den Tätern wichtig, sich im Einklang mit den in ihrer Wahrnehmung ausschlaggebenden Normen zu verhalten. Nicht Chaos und völlige Amoralität herrschen hier, sondern vielmehr eine in erschreckender Weise pervertierte Moral.

\section{Rortys Vokabularbegriff und das unterschätzte Potential rationaler Verständigung}

Mit ihrer Beschreibung der nationalsozialistischen Moral kommen Welzer und Gross dem sehr nahe, was Rorty mit seinem Begriff des Vokabulars zu erfassen versucht. Wie bereits erwähnt, besteht der zentrale Gedanke für Rorty darin, dass unsere Sicht der Welt immer von kulturell vermittelten Vorstellungen geprägt ist, die in je spezifischen Vokabularen gebündelt sind. Vokabulare in diesem Sinne gehen über natürliche Sprachen hinaus und umfassen auch Wertvorstellungen, kulturelle Traditionen und gesellschaftliche Verhaltensnormen. ${ }^{33}$ Der Vokabularbegriff dient Rorty dabei zunächst dazu, philosophische Letztbegründungsversuche zurückzuweisen. Wo diese den Anspruch erheben, nicht weiter hinterfragbare, von Raum und Zeit unabhängige Wahrheiten zu entdecken, beharrt Rorty auf der Kontingenz sprachlich vermittelter Vokabulare. So ausgeklügelt auch die Versuche sein mögen, dieser Kontingenz durch das Aufdecken unverrückbarer Wahrheiten zu entkommen, seien sie doch letztlich auf vorhandene Ausdrucksformen und damit auch auf vorhandene Vokabulare angewiesen. ${ }^{34}$ Diese Unhintergehbarkeit der Sprache und der durch sie vermittelten Deutungsmuster, so Rorty weiter, zeige sich in der Unmöglichkeit, sprachliche Aussagen an einer außersprachlichen Realität zu messen. Die Welt gebe nicht vor, welches Vokabular besser geeignet sei, sich ihr zu nähern, sie »spricht überhaupt nicht. Nur wir sprechen. Die Welt kann, wenn wir uns eine Sprache einprogrammiert haben, die Ursache dafür sein, daß wir Meinungen vertreten. Aber eine Sprache zum Sprechen kann sie uns nicht vorschlagen. Das können nur andere Menschen tun «. 35

Dient der Vokabularbegriff Rorty zunächst als erkenntnistheoretisches Argument, so nutzt er ihn bald auch in soziologischer Weise. Die Gesellschaft erscheint ihm dabei als unterteilt in Wir-Gruppen, die sich durch den Bezug auf unterschiedliche

32 Ebd., S. 161, 195 f.

33 Vgl. Rorty 1992 a, S. 23-27. Siehe zum Vokabularbegriff bei Rorty auch Tietz 2001 und Williams 2003.

34 Rorty 1982, S. 16.

35 Rorty 1992 a, S. 25. 
Vokabulare voneinander unterscheiden. ${ }^{36}$ Vor diesem Hintergrund erklärt sich dann auch Rortys Skepsis ob der Möglichkeiten rationaler Verständigung über die Grenzen derart bestimmter Wir-Gruppen hinaus. Wie eingangs ausgeführt, geht Rorty davon aus, dass es hierzu gemeinsam geteilter Grundprämissen bedürfe, von denen ausgehend dann in deduktiver Form eine rationale Einigung erzielt werden kann. Anders als etwa Habermas, der diese geteilten Prämissen in Form einer zunächst nicht weiter problematisierten »Lebenswelt « grundsätzlich für gegeben und notfalls für rekonstruierbar hält, bezweifelt Rorty die Existenz einer ausreichenden Menge solcher Gemeinsamkeiten über die Grenzen von Vokabularen hinweg. ${ }^{37}$

Besonders krass, so Rorty, zeige sich dies am Beispiel des Nationalsozialismus. Und in der Tat lassen sich die Überlegungen von Welzer und Gross im Sinne von Rortys Vokabularbegriff deuten, lässt sich die nationalsozialistische Moral als ein spezifisches Vokabular verstehen. Die Zeit der Weimarer Republik erscheint aus dieser Perspektive als ein Wettstreit unterschiedlicher Vokabulare: Demokratische Ideen konfligieren mit dem Faschismus, beide wiederum stehen dem Kommunismus entgegen, und quer dazu zeigt sich noch immer ein starkes Nachwirken monarchistischer Überzeugungen. Mit der Machtergreifung der Nationalsozialisten setzt dann, wie von Welzer und Gross beschrieben, ein Prozess ein, dessen Ziel es ist, die nationalsozialistische Moral als allein gültigen Referenzrahmen, als allein gültiges Vokabular zu etablieren. Alternative Vokabulare werden entweder für die Zwecke des Nationalsozialismus zurechtgestutzt und so schließlich integriert oder aber schlicht mit roher Gewalt verdrängt.

Nochmals zugespitzt findet dieser Prozess bei den an den "Judenaktionen « beteiligten Soldaten statt. Das für viele der Männer ungewohnte Umfeld und der gerade dadurch immer ausschließlichere Fokus auf die Bezugsgruppe der Kameraden und Vorgesetzten wirken dabei verstärkend. Zu Beginn zeigt sich bei vielen von ihnen noch Widerstreben ob der Aufgabe, mit ihren eigenen Händen Juden zu töten. Spürbar ist hier noch die frühere Sozialisation der Männer in einem Vokabular, das ihnen das Töten wehrloser Menschen zumindest anfangs noch als problematisch erscheinen lässt. Bald schon ändert sich dies jedoch, wie Welzer beobachtet: »Die Gruppen bildeten ihre eigenen Referenzrahmen aus und waren in diesem Sinn für ihre Mitglieder total - sie stellten ein weitgehend alternativloses Deutungsangebot für das bereit, was man wahrnahm und wie man sich zu verhalten hatte «. ${ }^{38}$

Die nun routinemäßig mordenden Männer haben sich zu diesem Zeitpunkt so vollständig mit dem auf Ausschluss gründenden Vokabular der Nationalsozialisten identifiziert, dass hier tatsächlich der von Rorty befürchtete Fall eintritt und Verständigung nicht mehr möglich ist. Zwar besteht noch immer eine minimale Überlappung zwischen dem Vokabular der Täter und dem ihrer Opfer, die sich daran zeigt, dass diese durchaus einfache Informationen austauschen. Allerdings ist es zu diesem Zeitpunkt für die Soldaten kaum noch begreiflich, warum ihre Opfer sie um

36 Rorty 1992 b.

37 Rorty 2001 c, S. 110-111; vgl. Habermas 1995 [1981], S. 185.

38 Welzer 2007, S. 263.

Leviathan, 40. Jg., 1/2012 
Erbarmen und Hilfe anflehen. In der Vorstellungswelt der Soldaten, in dem für sie nun entscheidenden Vokabular, tauchen die Opfer gar nicht mehr als Personen auf, denen gegenüber man zu Rechenschaft, geschweige denn zu Hilfe verpflichtet ist. Appelle der Opfer, die sich etwa auf allen Menschen qua Menschsein in gleicher Weise zustehende Rechte beziehen, laufen ins Leere, da das Vokabular der Täter eben gerade von einem Menschenbild geprägt ist, das die Gleichheit aller Menschen in radikalster Form negiert. ${ }^{39}$ Und nicht nur erreichen die Appelle der Opfer die Täter daher nicht, als Projektion ihrer eigenen Wahrnehmung deuten sie dann sogar noch den Schock und die Apathie ihrer Opfer als vermeintliche Gelassenheit und als Beleg für deren implizites Einverständnis mit ihrer Ermordung. ${ }^{40}$

$\mathrm{Zu}$ beobachten ist hier der Extremfall des stummen Aufeinanderprallens zweier Vokabulare, mit tödlichen Konsequenzen für die Opfer. Es sind solche Situationen, auf die Rorty mit seinem Vokabularbegriff aufmerksam machen möchte, Situationen, in denen die Gemeinsamkeiten zwischen zwei Kommunikationsteilnehmern so gering sind, dass jenseits von Trivialitäten keine Verständigung mehr möglich ist. Vollständig eingenommen von ihrem Vokabular, sind die Täter taub gegenüber Argumenten, die ihnen das dramatische Ausmaß ihrer Vergehen deutlich machen könnten. Doch so wichtig der Hinweis auf die immer bestehende Möglichkeit des Scheiterns rationaler Verständigung ist, so wenig überzeugt es, wenn Rorty diese Warnung vor dem Extremfall zum Anlass nimmt, das Scheitern rationaler Verständigung überhaupt zu propagieren. Was er hierbei nämlich aus dem Auge verliert, ist, dass dieses Scheitern eine Vorgeschichte hat, die das alltägliche Gelingen von Verständigung offenbart.

Ein solcher Perspektivenwechsel hin zu den Möglichkeiten rationaler Verständigung setzt jedoch voraus, dass man den von Rorty selbst nur vage bestimmten Begriff des Vokabulars stärker ausdifferenziert. Rorty bietet, wie sich im Folgenden zeigen wird, wichtige Ansatzpunkte hierfür, entwickelt diese selbst aber nicht systematisch weiter. So ist es (1) nötig, den dynamischen Charakter von Vokabularen stärker hervorzuheben. Rorty neigt dazu, Vokabulare als statisch zu begreifen, daher auch seine Vorstellung vom stummen Aufeinanderprallen zweier Vokabulare. Die einzig mögliche Veränderung besteht für ihn darin, durch einen kreativ-eruptiven Akt ein neues Vokabular zu schaffen. ${ }^{41}$ Wie sich am Beispiel des nationalsozialistischen Vokabulars zeigt, entwickeln sich Vokabulare jedoch fortwährend weiter. Dadurch entsteht immer wieder die Möglichkeit, zunächst nur geringe Gemeinsamkeiten sukzessive auszubauen und so die Basis für weitergehende Verständigung zu schaffen. Ähnlich wie Habermas weist auch Albrecht Wellmer auf diese dynamische Dimension von Verständigungsprozessen hin, wenn er betont, dass wir »beim Argumentieren häufig erst den Kontext erzeugen, durch den Argumente die

39 Avishai Margalit und Gabriel Motzkin weisen auf die Widersprüchlichkeiten in diesem Menschenbild hin, betonen aber auch dessen Bedeutung für die Erklärung der nationalsozialistischen Verbrechen; siehe Margalit, Motzkin 1997, S. 7-9.

40 Welzer 2007, S. 152 f.

41 Rorty 1992 b, S. 137; vgl. hierzu auch die Kritik von Iser 2008, S. 60-62. 
Kraft gewinnen, die sie haben können «. ${ }^{42}$ Das rasante Entstehen der »nationalsozialistischen Moral « hat die Schattenseite dieser Dynamik von Vokabularen gezeigt; ein Beispiel dafür, wie zunächst nur geringe Gemeinsamkeiten sukzessive ausgebaut werden können, ist die Aussöhnung der beiden »Erzfeinde « Deutschland und Frankreich nach $1945 .{ }^{43}$

Weiter zeigt sich (2) die Notwendigkeit, zwischen unterschiedlichen Ebenen von Vokabularen zu unterscheiden. Rorty selbst bezieht sich an einigen Stellen auf Michael Walzers Unterscheidung zwischen »dicken « und »dünnen « Wertungen und deutet an, dass auch vokabularbezogene Wir-Gemeinschaften in analoger Weise unterschieden werden können. »Dünne « Vokabulare sind demnach abstrakter als »dicke« Vokabulare, die stärker einem spezifischen Kontext verbunden sind. ${ }^{44}$ Rorty selbst führt diesen Punkt systematisch nicht weiter aus; tatsächlich hilft diese Unterscheidung aber, noch besser die Möglichkeiten der Überlappung zwischen Vokabularen zu verdeutlichen. So liegt es nahe anzunehmen, dass bestimmte, sehr basale »dünne "Vokabulare Bestandteil aller »dicken " Vokabulare sind. Rorty selbst gesteht in Anlehnung an Donald Davidson zu, dass es bestimmter Überlappungen bedürfe, um ein Vokabular überhaupt als solches verständlich zu machen. ${ }^{45}$ Völlige Inkommensurabilität, wie Rorty in früheren Schriften mit Blick auf Kuhns Paradigmenbegriff behauptet hatte, ist damit ausgeschlossen. ${ }^{46}$ Nimmt man also an, dass es ein Minimum an Gemeinsamkeiten immer schon geben muss, so ist die entscheidende Frage, ob dieses Minimum für eine rationale Verständigung ausreicht. Auch im Fall der Konfrontation zwischen dem deutschen Soldaten und seinem jüdischen Opfer ist ein Minimum an Gemeinsamkeit noch vorhanden; offenkundig reicht hier das Maß an Gemeinsamkeiten jedoch nicht für eine einigermaßen anspruchsvolle rationale Verständigung aus, die über den Austausch von basalen Informationen hinausgeht. Die logisch notwendige Überschneidung zwischen Vokabularen reicht mithin nicht immer aus, sie bietet prinzipiell jedoch zumindest einen Ansatzpunkt für rationale Verständigung.

Die Vorstellung von auf unterschiedlichen Ebenen angesiedelten »dicken « und »dünnen « Vokabularen verweist (3) zudem darauf, dass alle Menschen in ihrer Weltdeutung von mehr als nur einem Vokabular geprägt sind. Ein gläubiger Mensch etwa wird sowohl von einem globalen religiösen Vokabular als auch von dem spezifischen Vokabular seines nationalen Kontextes geprägt sein. Die daraus entstehenden Verflechtungen über Vokabulargrenzen hinweg eröffnen wiederum eine Reihe möglicher Anknüpfungspunkte für rationale Verständigung.

Schließlich zeigt sich am Beispiel des Nationalsozialismus (4) der von Rorty weitestgehend vernachlässigte politische Charakter von Vokabularen. Vokabulare er-

42 Wellmer 1993, S. 171.

43 Vgl. zum Aussöhnungsprozess zwischen Deutschland und Frankreich Kaelble 2003.

44 Rorty 2007 a, S. 44-46; vgl. Walzer 1996.

45 Rorty 2007 b, S. 53.

46 Zu Rortys Kuhn-Rezeption siehe Rorty 1979, S. 322-333; Rorty 1999. Vgl. hierzu auch Letson 1997, S. 87-106; Bernstein 2010 b. 
scheinen bei Rorty nicht nur als statisch, sondern zudem auch noch als nahezu unbezwingbare Naturgewalten. Zwar gesteht er dem Individuum zu, sich qua Reflexion bewusst für oder gegen ein bestimmtes Vokabular zu entscheiden; aufgrund ihres kollektiven Charakters scheinen sich Vokabulare einer zielgerichteten Veränderung jedoch zu entziehen. ${ }^{47}$ Hilfreich ist hier wiederum die Unterscheidung zwischen »dünnen « und »dicken " Vokabularen. Denn in der Tat erscheint es als besonders schwierig, ein weithin verbreitetes und weitestgehend unbewusst tradiertes Vokabular zielgerichtet zu verändern. Je detaillierter, je »dicker ", Vokabulare jedoch werden, je mehr sie sich auf einen konkreten sozialen Kontext beziehen, umso mehr zeigt sich auch, dass sie Folge spezifischer Machtverhältnisse sind - und ihrerseits neue Machtverhältnisse schaffen. Deutlich wird dies am Beispiel des nationalsozialistischen Vokabulars, das, wie schon beschrieben, binnen vergleichsweise kurzer Zeit mithilfe gezielter Maßnahmen als nahezu unanfechtbar etabliert wurde, um die Herrschaft des NS-Regimes zu stabilisieren. Es verwundert, dass gerade ein so politischer Denker wie Rorty diesem Aspekt so wenig Bedeutung zumisst. Denn so wie der Verweis auf den politischen Charakter von Vokabularen die dahinter stehenden Machtverhältnisse problematisiert, deutet er doch auch auf die Möglichkeiten der bewussten Gestaltung von Vokabularen hin. Die Nationalsozialisten haben diese mit katastrophalen Folgen ausgenutzt, nichts spricht jedoch grundsätzlich dagegen, dass Vokabulare auch auf Anschlussfähigkeit gegenüber anderen Vokabularen und damit wiederum auf das Ziel rationaler Verständigung hin ausgerichtet werden können. Der seit jeher die Wissenschaft besonders kennzeichnende weltweite Austausch ist ein Beispiel hierfür, ebenso wie die zunehmende globale Verbreitung völkerrechtlicher Konfliktlösungsmechanismen.

Zusammengenommen weisen diese Erweiterungen des Vokabularbegriffs auf die Vielzahl von Überlappungen zwischen Vokabularen und die daraus resultierenden Möglichkeiten rationaler Verständigung hin. Während Rorty diese Möglichkeiten von vornherein verwirft, wird das Gelingen rationaler Verständigung nun zu einer empirischen Frage, die sich nur im Einzelfall klären lässt. Dies wird auch noch einmal mit Blick auf die von Welzer untersuchten Täter deutlich. Begreift man deren Hineinwachsen in die nationalsozialistische » Tötungsmoral « als einen schrittweisen Prozess, so ist davon auszugehen, dass zumindest am Anfang dieses Prozesses die Männer kritischen Argumenten noch zugänglich waren. Zum einen war das nationalsozialistische Vokabular noch nicht zu ihrem ausschließlichen Bezugsrahmen geworden, zum anderen war der Prozess der inhaltlichen Umformung dieses Vokabulars vom Ausschluss bis hin zur Vernichtung der Juden noch nicht abgeschlossen. Nicht die Unfähigkeit, kritische Argumente zu verstehen, war zu diesem Zeitpunkt das Problem, sondern vielmehr die Tatsache, dass es kaum jemanden gab, der diese Argumente klar zur Sprache brachte. Damit ist noch nicht gesagt, dass die Männer sich durch die Reden eines überzeugten Humanisten von ihrem Handeln hätten abbringen lassen, wohl aber, dass ihnen dessen Argumente durchaus hätten verständlich gemacht werden können. 
Gerade dieser letzte Punkt ist für Rortys Absage an die Möglichkeiten rationaler Verständigung von besonderer Bedeutung. Vor dem Hintergrund seines jahrzehntelangen Kampfes gegen philosophische Letztbegründungsversuche scheint sich bei Rorty eine gewisse Dichotomie entwickelt zu haben: Auf der einen Seite stehen dabei unverrückbare Wahrheiten, deren Aufdecken eine unmittelbare Verhaltensänderung bewirkt, auf der anderen Seite die völlige Absage an jegliche Form rationaler Verständigung. Dabei übersieht Rorty zum einen das große Feld rationaler Verständigung, die sich auf gemeinsam geteilte Prämissen gerade im Bewusstsein von deren Kontingenz bezieht, die nicht absolute Wahrheit, aber doch rationale Rechtfertigung zum Ziel hat. Zum anderen scheint es ihm schwerzufallen, rationale Einsicht in ein Verhältnis zu anderen Handlungsmotiven zu stellen. ${ }^{48}$ Gute Gründe können eine Verhaltensänderung bewirken, sie können aber auch gegenüber anderen Motiven wie etwa dem Wunsch, anerkanntes Mitglied einer Gruppe zu sein, versagen. Von der Möglichkeit des Scheiterns einer solchen unmittelbaren Verhaltensänderung auf die Irrelevanz rationaler Verständigung zu schließen, bleibt aber unplausibel.

\section{Pluralismus von Vokabularen als Grundlage von Verständigung}

Abschließend möchte ich zwei Fragen beantworten: Erstens, worin besteht der Mehrwert einer Herangehensweise, die den Nationalsozialismus auch als Moralsystem sieht? Zweitens, welche politischen Schlussfolgerungen ergeben sich aus den hier entlang des Beispiels der NS-Moral entwickelten Erweiterungen von Rortys Vokabularbegriff?

Was die erste Frage betrifft, so ist zunächst einmal zu betonen, dass es natürlich keinesfalls darum gehen kann, den Nationalsozialismus in Gänze auf das hiermit verbundene Moralsystem zu reduzieren. Der Vorschlag ist vielmehr, die moralischen Komponenten des Nationalsozialismus ernster zu nehmen, um so das Verhalten überzeugter Nationalsozialisten rekonstruieren zu können. Unter etwas anderem Vorzeichen haben Lothar Fritze und Helmut König 2009 im »Leviathan « eine Diskussion zu eben diesem Thema geführt. Fritze hatte dabei vorgeschlagen, »totalitäre "Verbrecher als moralisch handelnde Akteure zu verstehen, deren moralische Urteile jedoch durch fehlgeleitete "außermoralische Überzeugungen « auf Abwege geraten seien. ${ }^{49}$ Die von Fritze vorgenommene Unterscheidung zwischen einerseits einer Moral, die von bestimmten fundamentalen Normen wie etwa dem Tötungsverbot geprägt ist, und andererseits »außermoralischen Überzeugungen « zeigt das Bemühen, am Kern einer universalistischen Moral festzuhalten und dennoch die Täter nicht in einen Bereich jenseits der Moral zu verschieben. ${ }^{50}$ Es wird jedoch, und hierauf hat auch Helmut König in aller Deutlichkeit hingewiesen, nicht klar, wie genau sich die von Fritze gedachte Grenze zwischen den »Grundnormen «

48 Rorty 2001 c, S. 107.

49 Fritze 2009, S. 13.

50 Ebd., S. 29.

Leviathan, 40. Jg., 1/2012 
der Moral und den "außermoralischen Überzeugungen « ziehen lassen soll. ${ }^{51}$ Neben den Schwierigkeiten einer klaren Abgrenzung legen die hier angestellten Überlegungen zudem nahe, dass es einen solchen fixen Kern der Moral nicht gibt. Folgt man Rorty, so sind auch die fundamentalsten Vorstellungen der Moral immer noch kontingent. Und wie die historischen Studien von Welzer und Gross zeigen, sind sie zudem keineswegs vor folgenschweren Manipulationen gefeit. Ohne dies hier vollständig ausführen zu können, scheint es sogar, dass diese Gefahr der Manipulation in die Grundstrukturen des modernen Moralverständnisses eingebaut ist. Denn so wie dieses sich zentral auf das Prinzip der Unparteilichkeit beruft, gibt es hier doch auch partikularistische Momente, die sich etwa an den besonderen Verpflichtungen von Eltern gegenüber ihren Kindern zeigen lassen oder auch an den Überlegungen kommunitaristisch inspirierter Autoren, die den besonderen Stellenwert nationaler Gemeinschaften betonen. ${ }^{52}$ Keineswegs soll damit gesagt sein, dass die partikulare NS-Moral eine logische Weiterentwicklung der modernen Moral ist. Wohl aber zeigt sich hier, und dies macht wohl das besonders Abgründige einer solchen Perspektive aus, die Fragilität noch der fundamentalsten moralischen Vorstellungen. Es ist im Übrigen wohl auch eben diese Sorge, die die von Fritze ausführlich zitierte Arendt mit Rorty verbindet. ${ }^{53}$

Wichtig scheint dabei gerade auch mit Blick auf die Auseinandersetzung zwischen Fritze und König die klare Trennung zwischen Beobachter- und Teilnehmerperspektive. Wenn König auf die »vollkommen irrsinnigen Behauptungen und Wahrnehmungen ${ }^{54}$ der NS-Täter hinweist, so lässt sich dies als eine Stellungnahme aus der Perspektive eines Teilnehmers an moralischen Diskursen verstehen. Eine solche kritische Stellungnahme ist jedoch nicht zu verwechseln mit einer primär soziologisch interessierten Perspektive auf Fragen der Moral. ${ }^{55}$ Versteht man Moral wie Welzer, Gross und wohl auch Fritze als handlungsleitenden normativen Referenzrahmen, so ist die Gültigkeit der in diesem Rahmen vorgebrachten moralischen Argumente zunächst zweitrangig. Entscheidend ist hier bloß die letztlich empirisch zu überprüfende Annahme, dass für die handelnden Akteure dieser normative Referenzrahmen ihrer spezifischen Gemeinschaft ausschlaggebend ist. Auch die Übertragung von Rortys Vokabularbegriff auf diese Debatte folgt zunächst einer solchen Beobachterperspektive. Anders als Rorty glaube ich aber, dass es möglich ist, die Einsichten der Beobachterperspektive in die Teilnehmerperspektive zu übertragen, und komme damit zur zweiten Frage: Welche politischen Schlussfolgerungen ergeben sich aus der vorgeschlagenen Ausdifferenzierung des Vokabularbegriffs?

51 König 2009, S. 45 f.

52 Vgl. Tugendhat 2001 sowie beispielhaft für entsprechende kommunitaristische Positionen Miller 2000 und Tamir 1993.

53 Vgl. dazu auch König 2009, S. 49 f.

54 Ebd., S. 42.

55 Vgl. hierzu analog Habermas' Ausführungen zum soziologischen Rechtsdiskurs einerseits und zum philosophischen Rechtsdiskurs andererseits (Habermas 1992, Kap. 2). 
Die ersten drei Erweiterungen des Vokabularbegriffs betonen die Möglichkeiten rationaler Verständigung. Eine solche Verständigung ist sich der Kontingenz ihrer Prämissen bewusst und kann daher nicht mit dem Anspruch verbunden werden, endgültige Wahrheiten zu entdecken. Sie ist aber doch durch den systematischen Austausch und die Überprüfung von Argumenten mehr als die von Rorty beschworene bloß affektiv motivierte Empathie. Die vierte Erweiterung, der Hinweis auf den politischen Charakter von Vokabularen, betont, dass Verständigung über Vokabulargrenzen hinweg ein inhärent politischer Gegenstand ist. Rorty selbst scheut davor zurück, sein Plädoyer für Demokratie und Menschenrechte aus seinen philosophischen Überlegungen herzuleiten, da er bereits durch eine solche Begründung einen Rückfall in die von ihm stets kritisierte Tradition philosophischer Letztbegründungsversuche befürchtet. Allenfalls ist er bereit, eine »vage Harmonie « zwischen seinem politischen und seinem philosophischen Denken zuzugestehen, der jedoch keinerlei argumentative Funktion zukomme. ${ }^{56}$

Dabei legt die Diskussion des Vokabularbegriffs sehr wohl politische Konsequenzen nahe: Wenn dieser in aller Deutlichkeit die Möglichkeit des Scheiterns rationaler Verständigung aufzeigt und wenn sich an einem Beispiel wie dem Nationalsozialismus die möglichen Folgen eines solchen Scheiterns zeigen, scheint es durchaus geboten, dem politisch entgegenzuwirken. Doch worin kann ein solches Entgegenwirken bestehen, will man zugleich Rortys Hinweis auf die Kontingenz aller Vokabulare ernst nehmen? Wie lässt sich eine positive Forderung formulieren, ohne damit in widersprüchlicher Weise eine nichtkontingente Position einzunehmen? Mir scheint, dass schon die so gestellte Frage in die falsche Richtung führt, indem hier ein falscher Widerspruch konstruiert wird. Tatsächlich ist es in keiner Weise widersprüchlich, eine Behauptung mit dem Anspruch allgemeiner Verbindlichkeit aufzustellen und zugleich deren Fallibilität anzuerkennen.

So lassen sich die hier angestellten Überlegungen zur weiteren Ausdifferenzierung des Vokabularbegriffs als Argument für die politische Schlussfolgerung nutzen, ein Neben- und Übereinander von Vokabularen institutionell abzusichern, das es dem Einzelnen ermöglicht, sein Handeln aus unterschiedlichen Perspektiven zu hinterfragen. Dahinter steht die Hoffnung, dass durch eine solche Vielzahl von Vokabularen an verschiedensten Stellen Möglichkeiten zur Verständigung geschaffen werden, die das Entstehen der von Rorty befürchteten stummen Konfrontation im Voraus verhindern. Ein solcher Vorschlag der institutionellen Absicherung eines Pluralismus von Vokabularen ist dabei eine Ergänzung zu den stärker auf das Individuum zielenden Konsequenzen, die Welzer aus seinen Überlegungen zur NS-Moral zieht.

Man erschauert, wenn Welzer am Ende seines Buchs über die nationalsozialistischen Täter fast schon resignierend festhält, dass der Mensch unter ungünstigen Umständen letztlich wohl zu allem fähig sei. Ein wenig überraschend betont er dann jedoch fast ausschließlich die Pflicht des Einzelnen, »sich über die Tragweite seiner Schlussfolgerungen jederzeit Rechenschaft abzulegen $«{ }^{57}$ Ohne Zweifel ist dieser Hinweis auf die individuelle Verantwortung von großer Bedeutung. Denn auch

56 Rorty 2001 b, S. 195.

57 Welzer 2007, S. 47.

Leviathan, 40. Jg., 1/2012 
wenn die Beobachtung der Selbstwahrnehmung der Täter als moralisch handelnde Akteure hilft, deren Verhalten zu erklären, enthebt eine solche Erklärung die Täter nicht ihrer individuellen Schuld. In der Wahrnehmung der Täter war die NS-Moral zum alleinig relevanten normativen Bezugspunkt geworden, und doch zeigen auch die Untersuchungen Welzers, dass die Dominanz der NS-Moral nicht zu verwechseln ist mit totaler Alternativlosigkeit. Im Gegenteil, die Weigerung Einzelner, sich an den Tötungen zu beteiligen, wie auch die frühere Sozialisation der Täter verweisen auf das - wenn auch eingeschränkte - Fortbestehen alternativer Deutungsmuster. Die so niemals gänzlich verlorengegangene Möglichkeit, die Verwerflichkeit des eigenen Handelns zu erkennen, begründet die individuelle Verantwortung der Täter für die Verbrechen, an denen sie teilgenommen haben.

Neben diesem Festhalten an der Verantwortung des Einzelnen verweisen Welzers eigene Überlegungen aber eben auch auf die Bedeutung gesellschaftlicher Institutionen für moralisches Handeln. War die Situation der Täter durch die Betonung von Kameradschaft, militärischer Pflicht und Verantwortung gegenüber der Heimat bewusst darauf ausgelegt, eigenverantwortliches Handeln zu unterdrücken, so lässt sich hieraus im Umkehrschluss die Forderung nach gesellschaftlichen Institutionen ableiten, die eben diese Art von Selbstreflexion befördern. Zumindest andeutungsweise vermutet dann auch Welzer, dass es Strukturen der Anerkennung bedarf, um eben diese Form hoch anspruchsvoller individueller Autonomie zu ermöglichen. ${ }^{58}$ Eine solche Anerkennung zu gewährleisten, so mein Argument, wäre Teil der Aufgabe, einen Pluralismus von Vokabularen institutionell abzusichern.

Der Zweck eines solchen Pluralismus bietet dabei auch ein Kriterium für die Kritik an einzelnen Vokabularen: Insofern das Ziel Verständigung ist, geraten partikularistische, auf Ab- und Ausgrenzung basierende Vokabulare unter einen besonderen Rechtfertigungsdruck, müssen sie doch zeigen, wie sie trotz ihres Partikularismus mit dem übergeordneten Ziel der Verständigung über Vokabulargrenzen hinweg vereinbar sind. Obschon es hier weiterer argumentativer Schritte bedürfte, laufen diese Überlegungen zumindest vorläufig auf die Grundprinzipien liberaler Demokratien hinaus und kommen damit letztlich doch wieder den politischen Forderungen von Rorty nahe. Anders als bei Rorty erscheint die liberale Demokratie nun jedoch nicht mehr als eine rein zufällige Laune, als maximal in einem Verhältnis »vager Harmonie « zu seinen Überlegungen zum Vokabularbegriff; vielmehr lässt sich ihre besondere Bedeutung als institutionelle Absicherung eines Pluralismus von Vokabularen erläutern. Ein solches Verständnis der besonderen Bedeutung liberaler Demokratie kann dabei auch und gerade überall dort als Maßstab der Kritik dienen, wo heutige Demokratien Tendenzen der Abschottung gegenüber alternativen Vokabularen zeigen, sei es nach innen im Beharren auf der Überlegenheit einer vermeintlichen nationalen Leitkultur gegenüber kulturellen Minderheiten, sei es nach außen in der Behauptung der unbezweifelbaren Überlegenheit der eigenen politischen und kulturellen Werte. Die Demokratie als politisches System ist mithin keinesfalls der fortwährenden kritischen Hinterfragung enthoben; im besten Fall er- 
möglicht sie jedoch gerade eine solche Hinterfragung, indem sie ein weites Spektrum möglicher Deutungen in Form alternativer Vokabulare sichert.

Zugleich, und auch dies übersieht Rorty trotz seiner jahrelangen Auseinandersetzung mit Habermas, ist die Demokratie aber auf Verständigungsprozesse angewiesen. Indem er die Möglichkeiten rationaler Verständigung verwirft, entleert Rorty auch die demokratischen Verfahren. Wie etwa von Chantal Mouffe kritisiert, scheint die Vorstellung in sich homogener Wir-Gemeinschaften Rorty zu einem harmonistischen Verständnis demokratischer Auseinandersetzungen zu verleiten. ${ }^{59}$ Wenn jedoch, wie hier argumentiert, die Vorstellung derart homogener Vorstellungen nicht aufrechtzuerhalten ist und zudem normativ auch nicht wünschenswert erscheint, müssen die Möglichkeiten substantieller Konflikte im Rahmen demokratischer Entscheidungsverfahren stärker in den Mittelpunkt rücken. Will man solche Entscheidungen nicht in Willkür oder gewaltsame Auseinandersetzungen abgleiten lassen, setzen sie jedoch, und dies scheint Rorty nicht hinreichend zu beachten, wiederum Verständigungsprozesse voraus. Denn worauf, wenn nicht auf dem Austausch von Argumenten, sollten demokratische Entscheidungen beruhen?

\section{Literatur}

Aly, Götz 2005. Hitlers Volksstaat. Raub, Rassenkrieg und nationaler Sozialismus. Frankfurt a. M.: Fischer.

Arendt, Hannah 1950. "The aftermath of Nazi rule", in Commentary 10, S. 342-353.

Auer, Dirk 2004. Politisierte Demokratie. Richard Rortys politischer Antiessentialismus. Wiesbaden: VS Verlag für Sozialwissenschaften.

Barry, Brian 1995. Justice as impartiality. Oxford: Oxford University Press.

Bernstein, Richard J. 2008. »Richard Rorty's deep humanism «, in New Literary History 39, 1, S. 13-27.

Bernstein, Richard J. 2010 a. The pragmatic turn. Cambridge: Polity Press.

Bernstein, Richard J. 2010 b. »The specter haunting multiculturalism ", in Philosophy \& Social Criticism 36, 3-4, S. 381-394.

Browning, Christopher R. 1999. Ganz normale Männer. Das Reserve-Polizeibataillon 101 und die »Endlösung « in Polen. Reinbek bei Hamburg: Rowohlt.

Fritze, Lothar 2009. »Moralische Rechtfertigung und außermoralische Überzeugungen. Sind stotalitäre Verbrechen` nur in einer säkularen Welt möglich? ", in Leviathan 37, 1, S. 5-33.

Goldhagen, Daniel 2000. Hitlers willige Vollstrecker. Ganz gewöhnliche Deutsche und der Holocaust. München: Goldmann.

Gross, Neil 2008. Richard Rorty. The making of an American philosopher. Chicago: The University of Chicago Press.

Gross, Raphael 2010. Anständig geblieben. Nationalsozialistische Moral. Frankfurt a. M.: S. Fischer.

Habermas, Jürgen 1992. Faktizität und Geltung. Frankfurt a. M.: Suhrkamp.

Habermas, Jürgen 1995 [1981]. Theorie des kommunikativen Handelns. Band 2: Zur Kritik der funktionalistischen Vernunft. Frankfurt a. M.: Suhrkamp.

Iser, Mattias 2008. Empörung und Fortschritt. Grundlagen einer kritischen Theorie der Gesellschaft. Frankfurt a. M.: Campus.

Kaelble, Hartmut 2003. »Die sozialen und kulturellen Beziehungen Frankreichs und Deutschlands seit 1945 «, in Aus Politik und Zeitgeschichte 3-4, S. 40-46.

59 Mouffe 2007, S. 115-117.

Leviathan, 40. Jg., 1/2012 
König, Helmut 2009. »Moral, Politik und totalitäre Verbrechen. Bemerkungen zu Lothar Fritze: Moralische Rechtfertigung und außermoralische Überzeugungen. Sind ttotalitäre Verbrechen` nur in einer säkularen Welt möglich? «, in Leviathan 37, 1, S. 34-51.

Koonz, Claudia 2004. The Nazi conscience. Cambridge: The Belknap Press of Harvard University Press.

Letson, Ben H. 1997. Davidson's theory of truth and its implications for Rorty's pragmatism. New York: Peter Lang.

Margalit, Avishai; Motzkin, Gabriel 1997. »Die Einzigartigkeit des Holocaust ", in Deutsche Zeitschrift für Philosophie 45, 1, S. 3-18.

Miller, David 2000. Citizenship and national identity. Cambridge: Polity Press.

Mouffe, Chantal 2007. Über das Politische. Wider die kosmopolitische Illusion. Frankfurt a. M.: Suhrkamp.

Özmen, Elif 2008. "Unparteilichkeit ", in Handbuch der Politischen Philosophie und Sozialphilosophie, hrsg. v. Gosepath, Stefan; Hinsch, Wilfried; Rössler, Beate, S. 1376-1380. Berlin: De Gruyter.

Ramberg, Björn 2000. "Rorty und die Werkzeuge der Philosophie ", in Die Renaissance des Pragmatismus, hrsg. v. Sandbothe, Mike, S. 127-166. Weilerswist: Velbrück Wissenschaft.

Rorty, Richard 1979. Philosophy and the mirror of nature. Princeton: Princeton University Press.

Rorty, Richard 1982. Consequences of pragmatism. Essays, 1972-1980. Minneapolis: University of Minnesota Press.

Rorty, Richard 1992 a. »Die Kontingenz der Sprache", in Kontingenz, Ironie und Solidarität, hrsg. v. Rorty, Richard, S. 21-51. Frankfurt a. M.: Suhrkamp.

Rorty, Richard 1992 b. "Die Kontingenz eines liberalen Gemeinwesen ", in Kontingenz, Ironie und Solidarität, hrsg. v. Rorty, Richard, S. 84-123. Frankfurt a. M.: Suhrkamp.

Rorty, Richard 1998. Against bosses, against oligarchies. A conversation with Richard Rorty. Charlottesville: Prickly Pear Pamphlets.

Rorty, Richard 1999. »Thomas Kuhn, rocks and the laws of physics «, in Philosophy and Social Hope, hrsg. v. Rorty, Richard, S. 175-197. New York: Penguin Books.

Rorty, Richard 2000 a. "Menschenrechte, Rationalität und Empfindsamkeit ", in Wabrheit und Fortschritt, hrsg. v. Rorty, Richard, S. 241-268. Frankfurt a. M.: Suhrkamp.

Rorty, Richard 2000 b. »Wilde Orchideen und Trotzki ", in Philosophie \& die Zukunft, hrsg. v. Rorty, Richard, S. 137-159. Frankfurt a. M.: Fischer.

Rorty, Richard 2001 a. »Erwiderung auf Hauke Brunkhorst ", in Hinter den Spiegeln. Beiträge zur Philosophie Richard Rortys, hrsg. v. Schäfer, Thomas; Tietz, Udo; Zill, Rüdiger, S. 162-165. Frankfurt a. M.: Suhrkamp.

Rorty, Richard 2001 b. »Erwiderung auf Thomas Schäfer ", in Hinter den Spiegeln. Beiträge zur Philosophie Richard Rortys, hrsg. v. Schäfer, Thomas; Tietz, Udo; Zill, Rüdiger, S. 194-200. Frankfurt a. M.: Suhrkamp.

Rorty, Richard 2001 c. »Erwiderung auf Udo Tietz «, in Hinter den Spiegeln. Beiträge zur Philosophie Richard Rortys, hrsg. v. Schäfer, Thomas; Tietz, Udo; Zill, Rüdiger, S. 107-113. Frankfurt a. M.: Suhrkamp.

Rorty, Richard 2007 a. "Justice as a larger loyalty ", in Philosophy as cultural politics. Philosophical papers IV, hrsg. v. Rorty, Richard, S. 42-55. Cambridge: Cambridge University Press.

Rorty, Richard 2007 b. Philosophy as cultural politics. Philosophical papers IV. Cambridge: Cambridge University Press.

Rorty, Richard; Mendieta, Eduardo 2006. Take care of freedom and truth will take care of itself. Interviews with Richard Rorty. Stanford: Stanford University Press.

Talisse, Robert B. 2007. A pragmatist philosophy of democracy. Communities of inquiry. New York: Routledge.

Tamir, Yael 1993. Liberal nationalism. Princeton: Princeton University Press.

Tietz, Udo 2001. »Das 'principle of charity< und die ethnozentristische Unterbestimmung der hermeneutischen Vernunft ", in Hinter den Spiegeln. Beiträge zur Philosophie Richard Rortys, hrsg. v. Schäfer, Thomas; Tietz, Udo; Zill, Rüdiger, S. 77-106. Frankfurt a. M.: Suhrkamp.

Tugendhat, Ernst 2001. "Partikularismus und Universalismus ", in Aufsätze 1992-2000, hrsg. v. Tugendhat, Ernst, S. 57-66. Frankfurt a. M.: Suhrkamp. 
University of California Irvine 2010. Richard Rorty born digital files, 1988-2003. http://dspace 1.nacs.uci.edu/ (Zugriff vom 15.09.2011)

Walzer, Michael 1996. Lokale Kritik - globale Standards. Hamburg: Rotbuch.

Wellmer, Albrecht 1993. "Wahrheit, Kontingenz, Moderne ", in Endspiele: Die unversöhnliche Moderne, hrsg. v. Wellmer, Albrecht, S. 157-177. Frankfurt a. M.: Suhrkamp.

Welzer, Harald 2007. Täter. Wie aus ganz normalen Menschen Massenmörder werden. Frankfurt a. M.: S. Fischer.

Williams, Michael 2003. »Rorty on knowledge and truth «, in Contemporary philosophy in focus, hrsg. v. Guignon, Charles; Hiley, David, S. 61-81. Cambridge: Cambridge University Press. 
Zusammenfassung: Für Richard Rorty ist der Nationalsozialismus ein Beispiel für das notwendige Scheitern rationaler Verständigung. Ich argumentiere hingegen, dass bei einer stärkeren Ausdifferenzierung des für Rorty zentralen Begriffs des "Vokabulars « die Vorgeschichte solchen Scheiterns auf die vielfältigen Möglichkeiten rationaler Verständigung verweist sowie auf die Notwendigkeit, diese institutionell abzusichern.

Stichworte: Rorty, Nationalsozialismus, nationalsozialistische Moral, Verständigung, Vokabularbegriff

\section{Richard Rorty, National Socialism and the possibilities of rational communication}

Abstract: Richard Rorty sees National Socialism as a prime example of the failure of rational communication. I argue, however, that a more nuanced understanding of what Rorty describes as » vocabularies " and a closer look at the history of failed communication reveals the manifold, possibilities of rational communication - as well as the need to protect these possibilities through adequate institutions.

Keywords: Rorty, National Socialism, National Socialist morality, communication, vocabulary

Dipl.-Politologe Daniel Jacob

Freie Universität Berlin

SFB 700 »Governance in Räumen begrenzter Staatlichkeit «

Binger Str. 40

14197 Berlin

E-Mail: daniel.jacob@fu-berlin.de 